\title{
A simplified method to quantitatively predict the effect of lenvatinib on hepatocellular carcinoma using contrast-enhanced ultrasound with perfluorobutane microbubbles
}

\author{
Yuji Eso, Shigeharu Nakano, Masako Mishima, Soichi Arasawa, Eriko Iguchi, Haruhiko Takeda, \\ Atsushi Takai, Ken Takahashi, Hiroshi Seno \\ Department of Gastroenterology and Hepatology, Graduate School of Medicine, Kyoto University, Kyoto, Japan \\ Correspondence to: Yuji Eso, MD, PhD. Department of Gastroenterology and Hepatology, Graduate School of Medicine, Kyoto University, Shogoin- \\ Kawaharacho 54, Sakyo-ku, Kyoto 606-8507, Japan. Email: yujieso@kuhp.kyoto-u.ac.jp.
}

\begin{abstract}
Contrast-enhanced computed tomography (CECT) is generally used to evaluate the response to treatment of hepatocellular carcinoma (HCC); however, CECT is unsuitable for the early prediction of therapeutic effects and frequent monitoring. We aimed to investigate the usefulness of our simplified method for the quantification of tumor vascularity using contrast-enhanced ultrasound (CEUS) with perfluorobutane microbubbles [Sonazoid ${ }^{\circledR}$ (GE Healthcare, Oslo, Norway)] to predict the therapeutic effect of lenvatinib. Among the 13 patients studied, nine who had more than a $20 \%$ reduction in tumor vascularity within 2 weeks of starting treatment experienced complete response or partial response at 8-12 weeks as assessed by CECT. In contrast, three patients without reductions and one patient with only a slight decrease in tumor vascularity had a poor response to lenvatinib. Quantitative assessment of tumor vascularity by our simplified CEUS-based method could be a useful predictor of therapeutic responses to lenvatinib in patients with HCC.
\end{abstract}

Keywords: Contrast-enhanced ultrasound (CEUS); hepatocellular carcinoma (HCC); lenvatinib; sonazoid; timeintensity curve analysis

Submitted Aug 13, 2020. Accepted for publication Jan 20, 2021.

doi: 10.21037/qims-20-965

View this article at: http://dx.doi.org/10.21037/qims-20-965

\section{Introduction}

Contrast-enhanced ultrasound (CEUS) allows continuous observation of intratumoral hemodynamics and its contrast pattern can be an essential clue for the differential diagnosis of focal hepatic lesions (1). CEUS with perfluorobutane microbubbles [Sonazoid $^{\circledR}$ (GE Healthcare, Oslo, Norway)] is useful not only for diagnosis of hepatic lesions but also as guidance for percutaneous biopsy and local ablation therapy because it can stably depict the outline of the tumor during the post-vascular phase (2-5). In addition, some reports have attempted to evaluate blood flow in hepatic tumors quantitatively using CEUS (6,7). However, to date, there is no widely accepted and commonly used CEUS-based method for quantitative assessment of tumor vascularity in clinical practice. This may be due to the complex calculations required for quantitative evaluation of blood flow, the need to install commercial software, or the difficulty of reproducible evaluation unless the examiner is proficient in CEUS.

If an accurate quantitative assessment of liver tumor vascularity can be made by CEUS, it may be possible to detect a decrease in tumor blood flow due to the therapeutic effects of molecular targeted agents (MTAs) without using contrast-enhanced computed tomography (CECT) or magnetic resonance imaging (MRI). Sorafenib, previously used as a first-line MTA for hepatocellular carcinoma (HCC), exhibited a low response rate and few reports have attempted to determine early therapeutic efficacy by 
CEUS $(8,9)$. Recently, lenvatinib (Lenvima ${ }^{\circledR}$, Eisai Co., Ltd., Tokyo, Japan) was approved as a novel first-line MTA for unresectable HCC based on the phase III REFLECT trial (10). Lenvatinib exerts antitumor activity by inhibiting angiogenesis through blockage of both vascular endothelial growth factor and fibroblast growth factor pathways (11). In fact, patients with HCC who experienced remarkable responses to lenvatinib show marked decreases in tumor vascularity by CECT scans (12). However, CECT is not suitable for early prediction of treatment response or frequent monitoring because overutilization of CECT increases medical costs and the patient's risk of both contrast media and radiation exposure.

In the present study, we performed CEUS on patients with HCC treated with lenvatinib and attempted to identify early therapeutic efficacy by quantitatively assessing tumor vascularity before and 1 to 2 weeks after treatment initiation. In addition, we aimed to develop a simplified method of quantitative assessment of tumor vascularity using the built-in quantification software on the ultrasound system in order for it to be performed easily and reproducibly by non-skilled sonographers.

\section{Methods}

In total, 43 patients with HCC received treatment with lenvatinib at Kyoto University Hospital (Kyoto, Japan) from March 2018 to July 2019. The diagnosis of HCC was based on CECT, MRI, and/or CEUS. We collected clinical data for these patients before treatment with lenvatinib, including treatment history and blood test results. The ethics committee of Kyoto University Hospital approved the study protocol (protocol number R2009), and we received informed consent from each participant.

\section{Sonazoid-enhanced ultrasound imaging}

The sonographic scans were performed with a LOGIQ E9 system (GE Healthcare, Milwaukee, WI, USA) using a convex probe. The dual-display function on the ultrasound system was used, providing a simultaneous display of the conventional B-mode and CEUS on a single monitor. For CEUS, one vial $(16 \mu \mathrm{g})$ of perfluorobutane was reconstituted with $2 \mathrm{~mL}$ of sterile water for injection. An intravenous bolus injection of the perfluorobutane solution was administered in a volume of $0.5 \mathrm{~mL}$, followed by immediately with a manual $10-\mathrm{mL}$ saline flush for approximately 3 seconds. To avoid disrupting the microbubbles, we selected a low-mechanical index $(0.2-0.25)$ and set the focus below the tumor of interest.

\section{Quantitative assessment of tumor vascularity by CEUS}

The quantitative assessment of tumor vascularity was performed with time-intensity curve (TIC) analysis using the LOGIQ E9 system. First, we selected one hyper-vascularized target tumor that could be stably observed within $10 \mathrm{~cm}$ from the body surface by CEUS and was considered suitable for TIC analysis, referring to the prior CECT and B-mode ultrasonography. Second, a video was recorded during the early vascular phase of CEUS (Figure $1 A$ ). Third, during TIC analysis mode, two regions of interest (ROI) were set at the most intensely imaged area in the tumor and the background liver area at the same depth, and then two TICs were displayed (Figure $1 B$ ). In order to make the method of quantifying tumor vascularity as simple and widely accepted as possible, we used the default ROI size of $5 \mathrm{~mm}$ without changing it from case to case. Fourth, a smoothing process was applied to the TICs. The starting point of the quantitative assessment was set from the junction of the two TICs where the curves start splitting into different curves, and the endpoint was set $15 \mathrm{~s}$ after the starting point, considering the time period that all patients can stably hold their breath (Figure $1 C$ ). Finally, several parameters, including the area under the curve (AUC), were automatically calculated from the TICs. The quantitative tumor vascularity was defined as the AUC of the tumor area minus that of the background liver area ( $\triangle \mathrm{AUC}$, Figure $1 D)$. To ensure the accuracy of the quantitative assessment, the TIC analysis was carried out in discussion with two or three examiners. Quantitative assessment of tumor vascularity was performed just before and 1 and/or 2 weeks after the start of treatment with lenvatinib.

\section{Protocol of treatment with lenvatinib}

The initial dose of lenvatinib was $8 \mathrm{mg}$ q.d. to patients weighing $<60 \mathrm{~kg}$ and $12 \mathrm{mg}$ once a day to those weighing $\geq 60 \mathrm{~kg}$. However, at the discretion of the attending physician, it was acceptable to start treatment with a reduced dose (from 12 to $8 \mathrm{mg}$, or from 8 to $4 \mathrm{mg}$ ) after obtaining informed consent. If unacceptable drug-related adverse events (AEs) occurred, the dose was reduced or treatment was interrupted. This dose reduction or temporary interruption of lenvatinib was maintained until the AEs were reduced to grade 1 or 2 . 

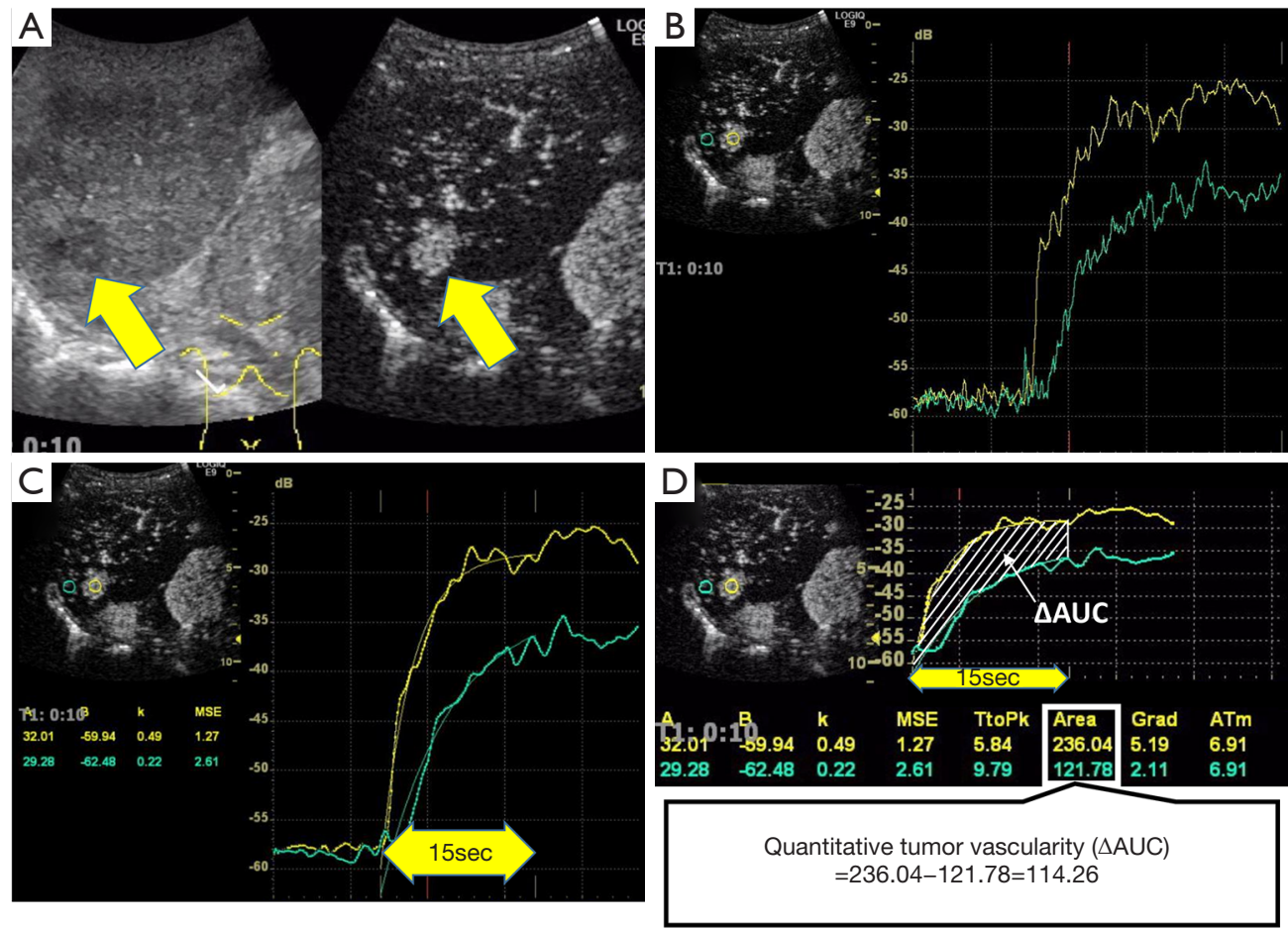

Figure 1 Quantitative assessment of tumor vascularity with Sonazoid-enhanced ultrasound. (A) A hypervascular tumor considered suitable for time-intensity curve (TIC) analysis was selected (yellow arrows). (B) Small regions of interest were set at the most intensely imaged area in the tumor (yellow circle) and the background liver area at the same depth (green circle). (C) The starting point of the quantitative assessment was set at the junction of the two TICs, and the endpoint was set $15 \mathrm{~s}$ after the starting point. (D) The quantitative tumor vascularity was defined as the area under the curve (AUC) of the tumor area minus that of the background liver area ( $\triangle \mathrm{AUC}$ ).

\section{Evaluation of therapeutic response}

Dynamic CECT scans were performed 8-12 weeks after treatment initiation to evaluate the therapeutic response to lenvatinib. The response to lenvatinib [complete response (CR), partial response (PR), stable disease (SD), or progressive disease $(\mathrm{PD})]$ was evaluated by investigators in accordance with the modified Response Evaluation Criteria in Solid Tumors (mRECIST) guidelines (13).

\section{Results}

\section{Baseline clinical characteristics of patients}

Schematic flowchart of the patients enrolled in this study is shown in Figure 2. Among the 43 patients who started treatment with lenvatinib, seven discontinued treatment within 2 months due to AEs or disease progression. Thirteen patients did not undergo CEUS and four patients only had extrahepatic tumors. Among the 19 patients who underwent CEUS, TIC analysis was unsuccessful in six of them because their tumors could not be detected clearly during the early vascular phase of CEUS due to their size or depth. Consequently, quantitative assessment of tumor vascularity before treatment and 1 and/or 2 weeks after treatment initiation was successfully performed in 13 patients, and their baseline characteristics are shown in Table 1. The present study included 10 males and three females (mean age, 69.7 years; age range, 50-85 years). One patient was administered lenvatinib as an initial treatment, while 12 patients had a treatment history for HCC.

\section{Therapeutic response to lenvatinib}

Among the 13 patients, seven patients experienced downtitration and one patient experienced a temporary interruption of lenvatinib treatment due to AEs. In the CECT imaging results of the 13 patients evaluated at $8-12$ weeks, CR was noted in one patient, $\mathrm{PR}$ in eight patients, SD in three patients, and PD in one patient following the mRECIST guidelines. 
Table 1 Baseline characteristics of patients who underwent time intensity curve analyses ( $\mathrm{n}=13)$

\begin{tabular}{|c|c|}
\hline Variables & Values \\
\hline Gender (male/female) & $10 / 3$ \\
\hline Body weight $(<60 \mathrm{~kg} / \geq 60 \mathrm{~kg})$ & $5 / 8$ \\
\hline ECOG PS (0/1) & $11 / 2$ \\
\hline BCLC stage $(B / C)$ & $7 / 6$ \\
\hline The number of intrahepatic tumors (range) & $5.38(3$ to 11$)$ \\
\hline Treatment history (treatment prior to lenvatinib), naïve/recurrence & $1 / 12$ \\
\hline Surgery & 1 \\
\hline Sorafenib & 1 \\
\hline Regorafenib & 1 \\
\hline AST (IU/L) & $49.1 \pm 25.6$ \\
\hline ALT (IU/L) & $29.0 \pm 15.6$ \\
\hline Platelets $\left(\times 10^{4} / \mu \mathrm{L}\right)$ & $14.6 \pm 5.78$ \\
\hline Serum albumin $(\mathrm{g} / \mathrm{dL})$ & $3.65 \pm 0.48$ \\
\hline Total bilirubin (mg/dL) & $0.87 \pm 0.25$ \\
\hline Prothrombin time (\%) & $88.3 \pm 11.8$ \\
\hline
\end{tabular}

Values are presented as mean \pm standard deviation (range) or number. ECOG PS, European Cooperative Oncology Group performance status; HBV, hepatitis B virus; HCV, hepatitis C virus; NBNC, non-B non-C; BCLC, Barcelona Clinic Liver Cancer, TACE, transarterial chemoembolization; AST, aspartate aminotransferase; ALT, alanine aminotransferase, ALBI, albumin-bilirubin; AFP, $\alpha$-fetoprotein; DCP, des- $\gamma$-carboxy prothrombin.

\section{Correlation between changes in tumor vascularity and response to lenvatinib}

The relationship between the early changes in tumor vascularity evaluated with CEUS and the responses to lenvatinib detected by CECT carried out after 8-12 weeks was investigated in all patients. As a result, all nine patients who experienced objective responses (CR or PR) showed marked decreases in $\triangle \mathrm{AUC}(\geq 20 \%)$ within 2 weeks after treatment initiation (Figure 3). In contrast, one case with
SD and 2/3 cases with PD showed slight increases in $\triangle \mathrm{AUC}$ after 1 week, and one case with PD showed a slight decrease in $\triangle \mathrm{AUC}$ after 2 weeks.

Two representative cases are shown in Figures 4,5. Figure 4 shows the case of a 67 -year-old male. He was initially treated with transarterial chemoembolization; however, a treatment switch to lenvatinib was planned due to disease progression. Although he weighed $79.0 \mathrm{~kg}$ weight and had a Child-Pugh score of 5 , lenvatinib was started at $8 \mathrm{mg} /$ day due to deterioration of his performance 


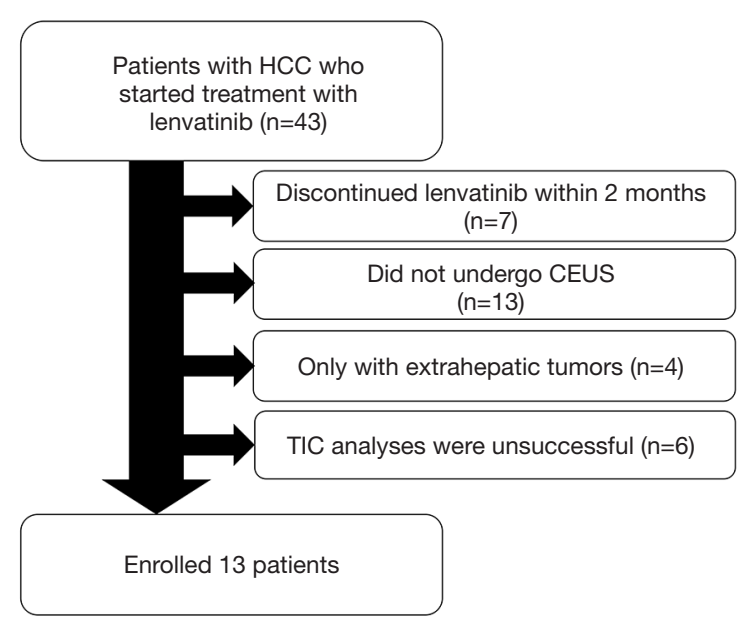

Figure 2 Schematic flowchart of the patients enrolled in this study. HCC, hepatocellular carcinoma; TIC, time-intensity curve; CEUS, contrast-enhanced ultrasound.

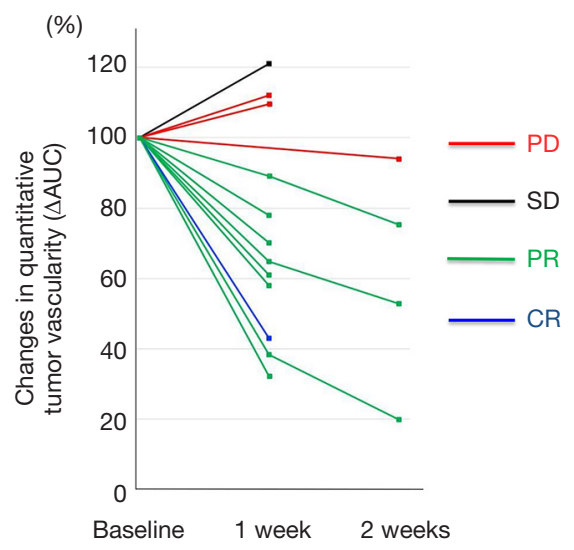

Figure 3 Correlation between early changes in quantitative tumor vascularity $(\triangle \mathrm{AUC})$ detected by Sonazoid-enhanced ultrasound and response to lenvatinib detected by CECT after 8-12 weeks. $\mathrm{PD}$, progressive disease; $\mathrm{SD}$, stable disease; $\mathrm{PR}$, partial response; $\mathrm{CR}$, complete response.

status. A hypervascular tumor, $2 \mathrm{~cm}$ in diameter, in segment five was considered suitable as a target lesion (Figure $4 A$ ). The baseline $\triangle \mathrm{AUC}$ evaluated one day before treatment initiation was 207.35 (Figure 4B). After treatment with lenvatinib for 1 week, the $\triangle \mathrm{AUC}$ decreased to 134.16 (64.7\% of initial value, Figure $4 C$ ). Furthermore, the $\triangle \mathrm{AUC}$ decreased to 109.42 (52.8\% of initial value) after 2 weeks (Figure 4D). He continued to receive treatment with $8 \mathrm{mg}$ until the CECT was performed after 9 weeks.
CECT revealed a marked decrease in tumor vascularity and indicated PR following mRECIST guidelines. Figure 5 shows the case of a 55-year-old woman who experienced multiple recurrences after liver resection. A $1.2-\mathrm{cm}-$ diameter tumor in segment three was selected (Figure 5A). The baseline $\triangle \mathrm{AUC}$ evaluated one day before treatment initiation was 110.79 (Figure 5B). Surprisingly, after treatment for 1 week with $12 \mathrm{mg} /$ day, the $\triangle \mathrm{AUC}$ decreased to 47.57 (42.9\% of initial value, Figure 5C). After 8 weeks of treatment CECT demonstrated the disappearance of enhancement in the arterial phase not only in the target lesion but also in other tumors. Thus, she was diagnosed as having CR (Figure 5D).

\section{Discussion}

For assessment of the treatment response to MTAs in patients with HCC, either the Response Evaluation Criteria in Solid Tumors 1.1 (RECIST 1.1) or mRECIST is recommended by the EASL guidelines (14). RECIST 1.1 is based on the measurement of the whole tumor, while mRECIST is based on measurement of the largest diameter of the arterialized tumor area (14). Both criteria need CECT or MRI scans; however, CECT or MRI at a very early stage, such as a few weeks after the start of treatment, is not suitable in terms of patient burden and costeffectiveness. Since, lenvatinib is an MTA that is associated with some AEs early in the course of treatment, such as hypertension, general malaise, and anorexia $(10,11,15)$; early prediction of the treatment effect contributes to an appropriate and individualized treatment plan and an improvement in patient motivation for treatment.

In the present study, we demonstrated that quantitative assessment of tumor vascularity by CEUS within 2 weeks of treatment with lenvatinib could predict therapeutic responses in patients with HCC. Among the 13 patients studied, the nine patients who showed marked decreases in quantitative tumor vascularity ( $\geq 20 \%$ ) experienced objective responses (CR or PR) as assessed by CECT performed 8-12 weeks after treatment initiation. In contrast, three patients without decreases in tumor vascularity and one patient with a slight decrease showed poor responses to lenvatinib (SD or PD).

Several methods have been proposed for the quantitative evaluation of tumor blood flow by contrast ultrasound. Shiozawa et al. previously reported the utility of arrival time parametric imaging using CEUS in predicting treatment effects of sorafenib. They measured the mean arrival time 

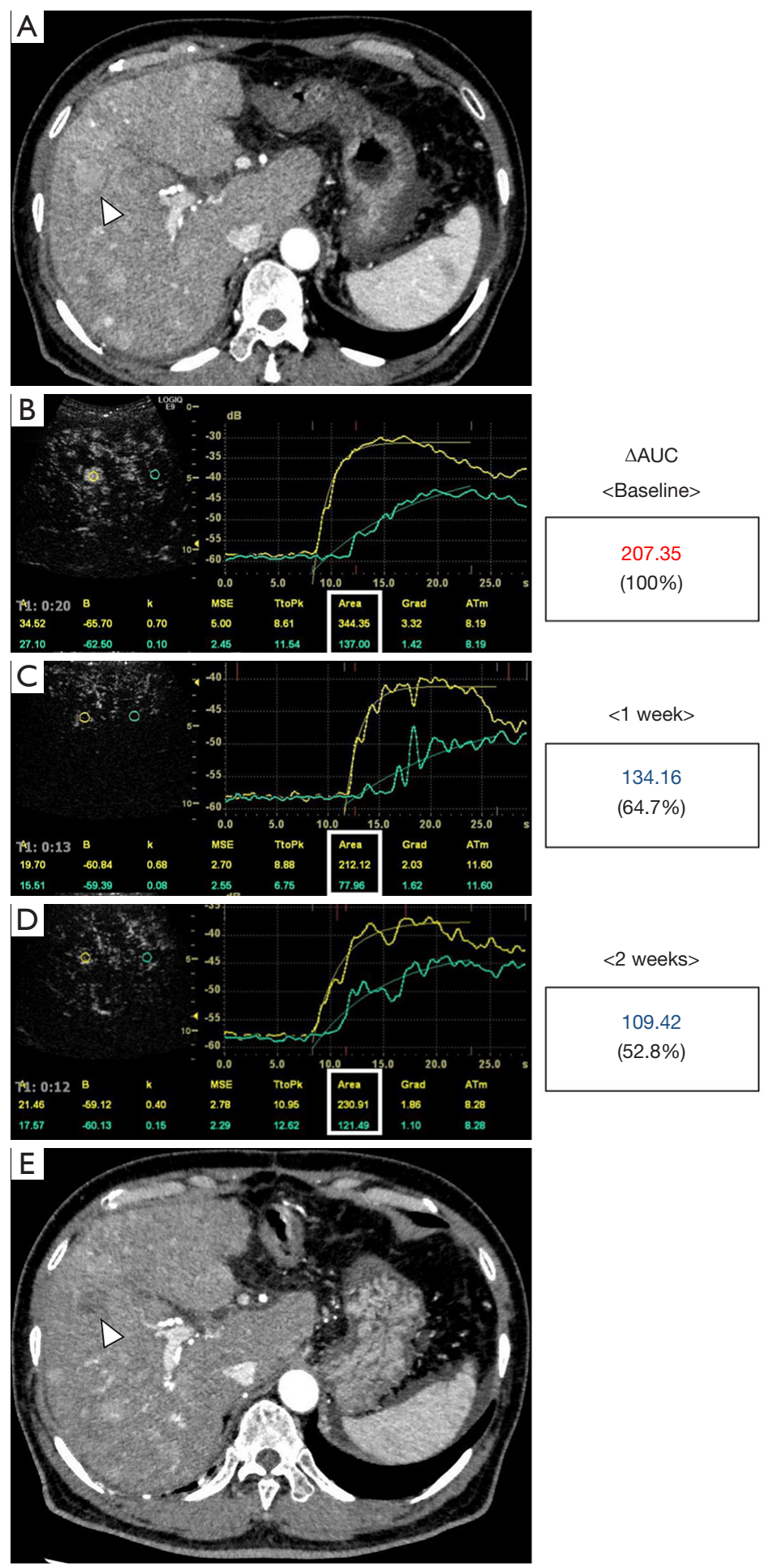

Figure 4 Case of a 67-year-old male patient. (A) A 2-cm diameter hypervascular tumor in segment five was considered suitable as a target lesion (arrowhead). (B) The baseline $\triangle \mathrm{AUC}$ evaluated before treatment initiation was 207.35. (C) $\Delta \mathrm{AUC}$ decreased to 134.16 (64.7\% of initial value) after 1 week of treatment. (D) $\triangle \mathrm{AUC}$ decreased to 109.42 (52.8\% of initial value) after 2 weeks. (E) CECT performed after 9 weeks showed marked decrease in tumor vascularity (arrowhead). 

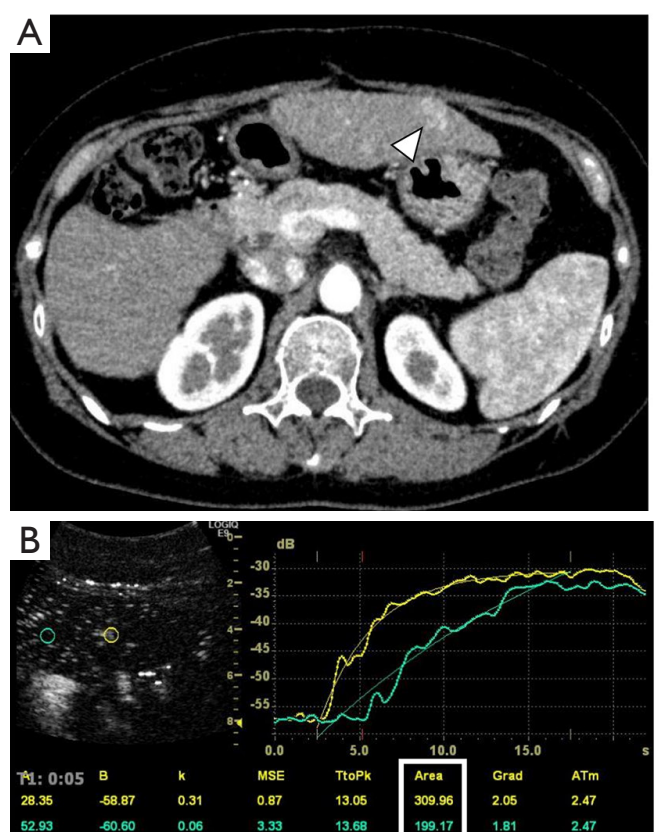

$\triangle \mathrm{AUC}$

$<$ Baseline $>$
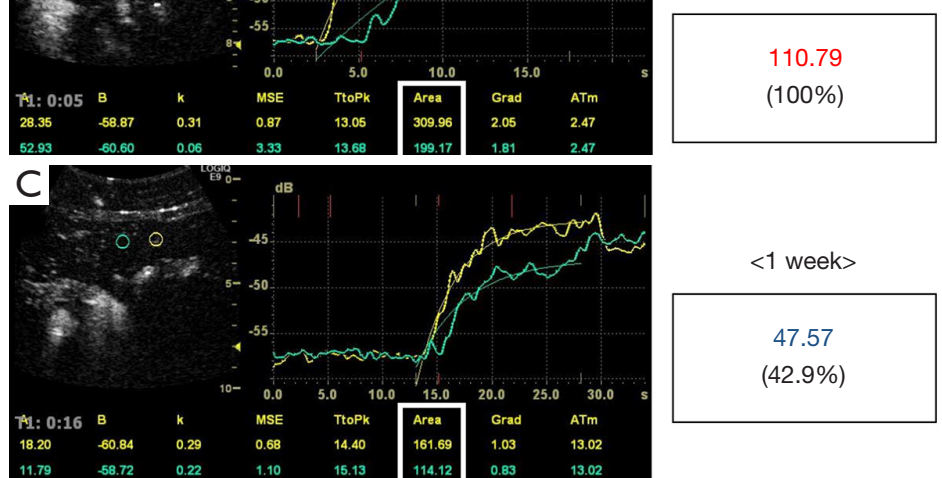

$<1$ week $>$
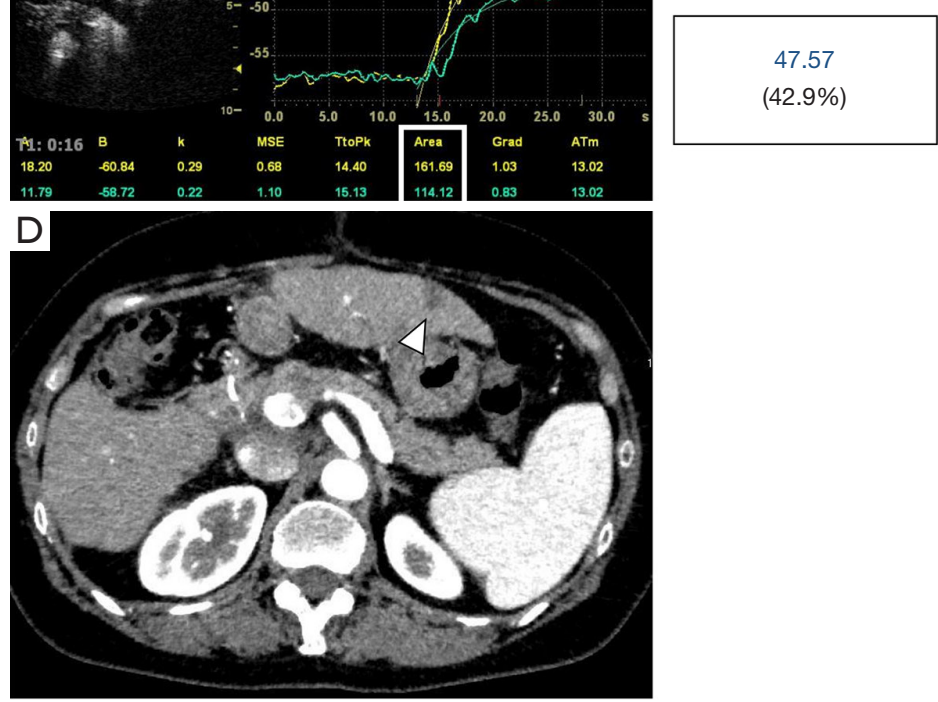

Figure 5 Case of a 55-year-old female patient. (A) A 1.2-cm diameter hypervascular tumor was detected in segment three by CECT (arrowhead). (B) The baseline $\triangle \mathrm{AUC}$ evaluated before treatment initiation was 110.79 . (C) $\triangle \mathrm{AUC}$ decreased to 47.57 (42.9\% of initial value) after 1 week of treatment. (D) Computed tomography performed after 8 weeks showed marked decrease in tumor vascularity (arrowhead).

of the contrast agent in the target lesion from a reference point (9). Their method requires setting an appropriate reference point not in the background liver but within the vessel (such as an intrahepatic artery near the tumor), which could lead to discrepancies in the results due to the examiner. In addition, since the reference point is set in the artery, it may be affected by treatment-induced hemodynamic changes. Sugimoto et al. (16) and Kuroda et al. (7) reported the methods using the rate of change for total AUC during "wash-in". Their methods are similar to ours. However, they did not set a reference point; thus, their data may still be affected by hemodynamic arrival changes. 
In our method, the ROI in the tumor and background liver can be set to an appropriate position while repeatedly checking the video images, which reduces the possibility of blurred results even for inexperienced sonographers. Since the calculation method is also simple, it could be widely accepted. However, it should be noted that the change in tumor vascularity of a single tumor detected by CEUS does not necessarily apply to all other tumors.

Overall, our findings have potentially important clinical implications for the quantitative assessment of tumor vascularity by CEUS as a predictor of therapeutic responses to lenvatinib in patients with HCC. However, our study has several limitations. First, in some patients (6 out of 19 in this study), the quantitative assessment of tumor vascularity was difficult due to the small size or depth from the liver surface. Second, our study was a single-center design and due to a small sample size, it was difficult to conduct a comparative analysis of response and non-response cases. Thus, caution should be exercised in interpreting our initial experience. In addition, in order to demonstrate the superiority and simplicity of our quantification method, further studies are required to compare with existing other commercial software packages.

\section{Conclusions}

In conclusion, we demonstrated that quantitative assessment of tumor vascularity by our simplified CEUS-based simplified method could be a useful predictor of therapeutic responses to lenvatinib in patients with HCC.

\section{Acknowledgments}

The authors would like to thank Hitomi Eso and our family and the medical staff at Kyoto University Hospital for their support and assistance with data collection. We would also like to thank Editage (http://www.editage.jp) for English language editing.

Funding: This research was supported by The Ministry of Education, Culture, Sports, Science and Technology (MEXT) KAKENHI Grant Number 19K17458.

\section{Footnote}

Conflicts of Interest: All authors have completed the ICMJE uniform disclosure form (available at http://dx.doi. org/10.21037/qims-20-965). The authors have no conflicts of interest to declare.
Ethical Statement: The study was approved by the ethics committee of Kyoto University Hospital (protocol number R2009), and informed consent was obtained from all the patients who participated in this study. All experiments were performed according to Declaration of Helsinki guidelines and regulations.

Open Access Statement: This is an Open Access article distributed in accordance with the Creative Commons Attribution-NonCommercial-NoDerivs 4.0 International License (CC BY-NC-ND 4.0), which permits the noncommercial replication and distribution of the article with the strict proviso that no changes or edits are made and the original work is properly cited (including links to both the formal publication through the relevant DOI and the license). See: https://creativecommons.org/licenses/by-nc-nd/4.0/.

\section{References}

1. Hatanaka K, Kudo M, Minami Y, Ueda T, Tatsumi C, Kitai S, Takahashi S, Inoue T, Hagiwara S, Chung H, Ueshima K, Maekawa K. Differential diagnosis of hepatic tumors: value of contrast-enhanced harmonic sonography using the newly developed contrast agent, Sonazoid. Intervirology 2008;51 Suppl 1:61-9.

2. Kudo M, Hatanaka K, Maekawa K. Newly developed novel ultrasound technique, defect reperfusion ultrasound imaging, using sonazoid in the management of hepatocellular carcinoma. Oncology 2010;78 Suppl 1:40-5.

3. Park HS, Kim YJ, Yu MH, Jung SI, Jeon HJ. Realtime contrast-enhanced sonographically guided biopsy or radiofrequency ablation of focal liver lesions using perflurobutane microbubbles (sonazoid): value of Kupfferphase imaging. J Ultrasound Med 2015;34:411-21.

4. Eso $Y$, Takai A, Takeda H, Matsumoto T, Lee M, Inuzuka T, Takahashi K, Ueda Y, Marusawa H, Seno H. Sonazoidenhanced ultrasonography guidance improves the quality of pathological diagnosis in the biopsy of focal hepatic lesions. Eur J Gastroenterol Hepatol 2016;28:1462-7.

5. Eso Y, Kou T, Nagai H, Kim YH, Kanai M, Matsumoto S, Mishima M, Arasawa S, Iguchi E, Nakamura F, Takeda H, Takai A, Takahashi K, Ueda Y, Muto M, Seno H. Utility of ultrasound-guided liver tumor biopsy for nextgeneration sequencing-based clinical sequencing. Hepatol Res 2019;49:579-89.

6. Shiozawa K, Watanabe M, Sumino Y. Evaluation of the hemodynamic status of focal hepatic lesions $20 \mathrm{~mm}$ or less in diameter by contrast-enhanced ultrasonography using 
Sonazoid. Intervirology 2009;52:213-22.

7. Kuorda H, Abe T, Fujiwara Y, Okamoto T, Yonezawa M, Sato H, Endo K, Oikawa T, Sawara K, Takikawa Y. Change in arterial tumor perfusion is an early biomarker of lenvatinib efficacy in patients with unresectable hepatocellular carcinoma. World J Gastroenterol 2019;25:2365-72.

8. Moschouris H, Malagari K, Gkoutzios P, Kalokairinou M, Stamatiou K, Chatzimichail K, Kornezos I, Karagiannis E, Kiltenis M, Papadaki MG. Intermediate and advanced hepatocellular carcinoma treated with the antiangiogenic agent sorafenib. Evaluation with unenhanced and contrastenhanced ultrasonography. Med Ultrason 2012;14:87-94.

9. Shiozawa K, Watanabe M, Ikehara T, Shimizu R, Shinohara M, Igarashi Y, Sumino Y. Evaluation of sorafenib for advanced hepatocellular carcinoma with low $\alpha$-fetoprotein in arrival time parametric imaging using contrast-enhanced ultrasonography. J Med Ultrason (2001) 2017;44:101-7.

10. Kudo M, Finn RS, Qin S, Han KH, Ikeda K, Piscaglia F, Baron A, Park JW, Han G, Jassem J, Blanc JF, Vogel A, Komov D, Evans TRJ, Lopez C, Dutcus C, Guo M, Saito K, Kraljevic S, Tamai T, Ren M, Cheng AL. Lenvatinib versus sorafenib in first-line treatment of patients with unresectable hepatocellular carcinoma: a randomised phase
3 non-inferiority trial. Lancet 2018;391:1163-73.

11. Eso Y, Marusawa H. Novel approaches for molecular targeted therapy against hepatocellular carcinoma. Hepatol Res 2018;48:597-607.

12. Takeda H, Nishijima N, Nasu A, Komekado H, Kita R, Kimura T, Kudo M, Osaki Y. Long-term antitumor effect of lenvatinib on unresectable hepatocellular carcinoma with portal vein invasion. Hepatol Res 2019;49:594-9.

13. Lencioni R, Llovet JM. Modified RECIST (mRECIST) assessment for hepatocellular carcinoma. Semin Liver Dis 2010;30:52-60.

14. Tovoli F, Renzulli M, Granito A, Golfieri R, Bolondi L. Radiologic criteria of response to systemic treatments for hepatocellular carcinoma. Hepat Oncol 2017;4:129-37.

15. Eso $\mathrm{Y}$, Nakano S, Mishima M, Arasawa S, Iguchi E, Nakamura F, Takeda H, Takai A, Takahashi K, Taura $\mathrm{K}$, Seno H. Dose Intensity/Body Surface Area Ratio is a Novel Marker Useful for Predicting Response to Lenvatinib against Hepatocellular Carcinoma. Cancers (Basel) 2019;12:353-60.

16. Sugimoto K, Moriyasu F, Saito K, Rognin N, Kamiyama N, Furuichi Y, Imai Y. Hepatocellular carcinoma treated with sorafenib: early detection of treatment response and major adverse events by contrast-enhanced US. Liver Int 2013;33:605-15.
Cite this article as: Eso Y, Nakano S, Mishima M, Arasawa S, Iguchi E, Takeda H, Takai A, Takahashi K, Seno H. A simplified method to quantitatively predict the effect of lenvatinib on hepatocellular carcinoma using contrast-enhanced ultrasound with perfluorobutane microbubbles. Quant Imaging Med Surg 2021;11(6):2766-2774. doi: 10.21037/qims-20-965 